\title{
Efficacy of Standardized Rehabilitation in the Treatment of Diastasis Rectus Abdominis in Postpartum Women
}

\author{
Jing $\mathrm{Hu}^{1}, *$ \\ Juanfen $\mathrm{Gu}^{1}{ }^{\prime} *$ \\ Zhiyan $\mathrm{Yu}^{\prime}$ \\ Xianxu Yang' \\ Jian Fan' \\ Lingying You' \\ Qinyan Hua' \\ Yuting Zhao' \\ Yuerong Yan' \\ Weiwei $\mathrm{Bai}^{2}$ \\ Zipeng $\mathrm{Xu}^{3}$ \\ Lina You' \\ Chaobo Chen $\mathbb{1}^{3,4}$ \\ 'Department of Postpartum \\ Rehabilitation Center, Xishan People's \\ Hospital of Wuxi City, Wuxi, Jiangsu, \\ People's Republic of China; ${ }^{2}$ Department \\ of Ultrasound, Xishan People's Hospital \\ of Wuxi City, Wuxi, Jiangsu, People's \\ Republic of China; ${ }^{3}$ Department of \\ General Surgery, Xishan People's \\ Hospital of Wuxi City, Wuxi, Jiangsu, \\ People's Republic of China; ${ }^{4}$ Department \\ of Immunology, Ophthalmology and ORL, \\ Complutense University School of \\ Medicine, Madrid, 28040, Spain \\ *These authors contributed equally to \\ this work
}

Correspondence: Lina You; Chaobo Chen Tel +86 I38I2052853; +86 I358500083 I Email 21721140@qq.com;

bobol9820106@gmail.com
Purpose: The incidence of diastasis rectus abdominis (DRA) in parturients is continuously increasing, which may cause uncomfortable and affect the quality of life. The present study aims to retrospectively summarize the experience and efficacy in the treatment of DRA via standardized rehabilitation procedures in Eastern China.

Methods: This retrospective study included the parturients with DRA admitted to the Xishan People's Hospital of Wuxi between January 2017 and May 2021. Patients were separated into standardized rehabilitation group (SR) and non-standardized rehabilitation group (non-SR). The outcomes were the change in rectus abdominis separation and Physical Functioning Scale (PFS). Measurement data were compared between the two groups, and multivariate linear regression was used to analyze the factors associated with the standardized rehabilitation process. $\mathrm{P}$ values $<0.05$ were considered statistically significant.

Results: Among a total of 294 patients with DRA who were included in the study, 171 patients were treated with SR (SR), and the other 123 patients were treated without SR process (non-SR). Compared with non-SR, the separation of the rectus abdominis was significantly reduced in SR after standardized rehabilitation treatment ( $\mathrm{p}$ value $<0.0001$ ). The multiple linear regression model analysis results suggested that standardized rehabilitation was an independent factor influencing the prognosis of DRA in parturients $(p<0.0001)$. In addition, the quality of life of the study group was significantly improved $(p<0.0001)$. Conclusion: Standardized rehabilitation method revealed high efficiency in treating DRA in postpartum women and could improve the quality of life of parturients.

Keywords: diastasis rectus abdominis, postpartum women, rehabilitation, Physical Functioning Scale

\section{Introduction}

Diastasis rectus abdominis (DRA) is characterized by an increased distance between the rectus abdominis on both sides of the linea alba in pregnant and postpartum women. ${ }^{1,2}$ The incidence of DRA, which is about $46.5 \%$ to $100 \%$ in the middle and late stages of pregnancy and approximately $30 \%$ to $70 \%$ after delivery, ${ }^{3-5}$ is continuously increasing in China. ${ }^{6}$ The diagnostic criteria for DRA are that one or more points of the linea alba are more than $2 \mathrm{~cm}$ separation, including the level of the umbilicus or $4.5 \mathrm{~cm}$ above and below, or the midline bulge is visible with force. ${ }^{7,8}$ Parturients often have different degrees of DRA during the third trimester. ${ }^{6}$ If ineffectively treated or left without treatment, they 
can end up with long-term severe DRA. ${ }^{9}$ Not only does the abdomen swell and affect the appearance, but it may also cause the instability of the spine, resulting in lower back pain and affecting the quality of life. What's more serious is that some severe patients will eventually need surgical treatment to relieve clinical symptoms. ${ }^{10,11}$ However, when DRA is timely treated, the symptoms can be immediately relieved and postpartum women's quality of life be improved as well. ${ }^{2,12}$ Therefore, effective treatment is very important to improve the separation of rectus abdominis after delivery.

Currently, there are many methods for the treatment of patients with postpartum DRA, such as restraint band assistance, ${ }^{3}$ exercises for enhancing the pelvic floor muscles ${ }^{13}$ and/or abdominal binding, ${ }^{14}$ electrical stimulation therapy, ${ }^{15}$ acupuncture treatment in traditional Chinese medicine, ${ }^{16}$ and surgical treatment. ${ }^{10}$ Surgery is an effective method for treating DRA. However, it is not considered as an optimal choice, because it is a traumatic treatment method that may cause complications such as postoperative scars (even if minimally invasive surgery is used), wound infection, patch rejection, postoperative adhesions, and other complications. ${ }^{11,17}$ Moreover, if postpartum women need another abdominal surgery in the long term, it may be affected by the abdominal surgical repair. Furthermore, available methods for treatment of postpartum DRA are still controversial, and there is a lack of effective standardized non-surgical treatment programs. Previous study has reported that Chinese medicine-acupuncture can effectively ameliorate DRA. ${ }^{16}$ Nonetheless, this method is difficult to master, it requires a long learning cycle, and its promotion is very slow. Therefore, we have created this new systematic and standardized nonsurgical method for the treatment of DRA, which is based on the traditional Chinese medicine massage technique combined with the practical application of muscle and movement systems.

Traditional Chinese medicine acupuncture has a good effect on pain and body discomfort, ${ }^{18}$ while electrophysiology has a good physical therapy effect on muscles. ${ }^{15,19}$ Taking advantage of these two characteristics, Standardized Rehabilitation process is a complete systematic treatment mode, including 40 minutes of manual massage (Part 1) and 30 minutes of treatment with electrophysiological equipment (Part 2) (Figure 1). The standardized rehabilitation process was completed once every other day, and the entire standardized rehabilitation was performed 10 times before completion. Although, some methods have been applied in clinical practice over the five years, with good effects and social benefits for postpartum women with DRA. ${ }^{12,20}$ However, our proposed approach is easier to learn and use (Figure S1-S19). During the past three years, the quality of life of postpartum women with DRA was significantly improved after being treated by standardized treatment methods. We have obtained good social feedback as well. Therefore, the formulation of standardized rehabilitation treatment methods could benefit DRA patients, and we hope to benefit more patients.

The purpose of this study was to retrospectively summarize the treatment experience and efficacy of DRA in postpartum women who received standardized rehabilitation treatment in a clinical hospital in Eastern China between January 2017 and May 2021. Simultaneously, this study may help medical staff and rehabilitation physiotherapists who are engaged in postpartum rehabilitation in other clinical hospitals, assist in alleviating the symptoms of DRA patients, and improving the quality of life.

\section{Materials and Methods Study Design and Subjects}

This retrospective study included all the patients with diastasis rectus abdominis admitted at Xishan People's Hospital of Wuxi City between January 2017 and May 2021. This study was approved by the Ethics Committee of Xishan People's Hospital of Wuxi City. The requirement for informed consent was waived. This study strictly kept the patients' information confidential. The study complied with the Declaration of Helsinki.

Inclusion criteria were: 1) 22-36 years; 2) parturients, including caesarean section; 3) body mass index (BMI) $\leq 29 \mathrm{~kg} / \mathrm{m}^{2}$; 4) diagnosed with DRA between 3-6 months after delivery. ${ }^{2,21}$

Exclusion criteria were: 1) patients with incomplete data or missing follow-up data; 2) patients with any heart or respiratory disease, including excessive coughing and sneezing; 3) patients with any kind of pelvic or abdominal surgery.

\section{Data Collection}

The study group has completed the standardized rehabilitation treatment. Data collection and follow-up were carried out for both study and the control group during the same period. Collected data included age (year), weight $(\mathrm{kg})$, numbers of pregnancies, BMI $(\mathrm{kg} / \mathrm{m} 2)$, natural delivery, cesarean section, diabetes, hypertension, chronic constipation, abdominal pain, 


\section{Standardized rehabilitation for non-surgical treatment of DRA}

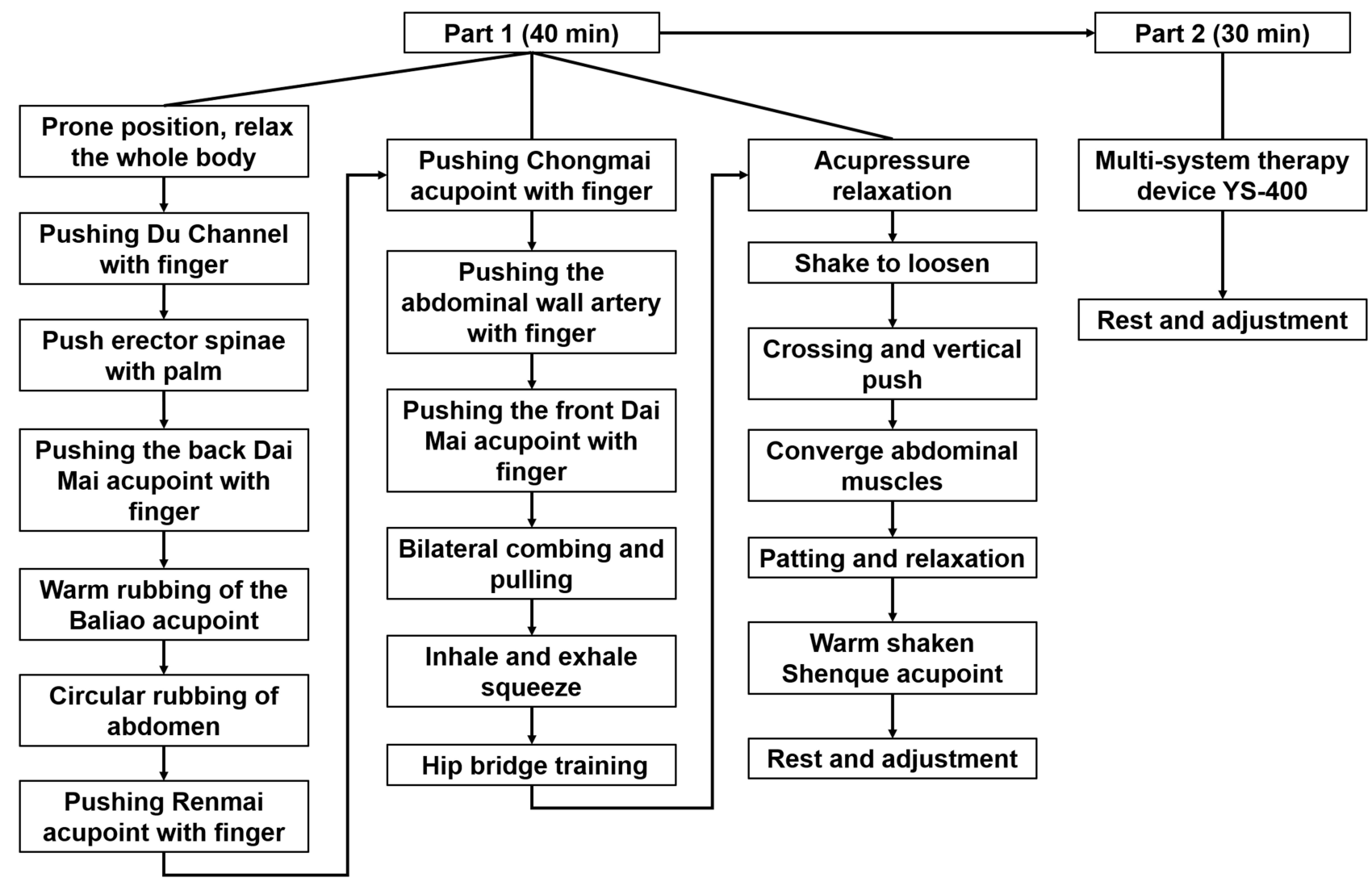

Figure I Standardized Rehabilitation. A representative process, which is promoted by a fixed step-by-step manual massage combined with the treatment of electrophysiological equipment.

pelvic pain, and low back pain, the inner edge of DRA and PFS from both groups. Both the study and the control groups were followed up for more than 3 months.

\section{Outcome Measures}

Each value was measured and calculated by doctors and nurses in the same group. The distance between the inner edges of the rectus abdominis was detected at the level of the umbilicus and $4.5 \mathrm{~cm}$ above/below the umbilicus by using a B-ultrasound probe, which is very accurate. Each measurement point of postpartum women was marked using soluble markers to ensure the standardization of repeated measurements.

\section{Physical Functioning Scale (PFS-10)}

The Physical Functioning Scale (PFS-10) is a self-reported health measurement tool developed in the United States using data from medical outcome research and applying it to patients with acute and chronic diseases. ${ }^{22}$ PFS-10 includes 10 items and is used to assess the degree of health-related limitations in physical functions. These items are scored on a 3-point Likert scale ( $1=$ very limited, $2=$ slightly limited, $3=$ not limited at all), which has been proven to be reliable and valid in previous studies. ${ }^{23,24}$ Based on this measurement, we compiled the data before and after the treatment of standardized rehabilitation for performing the statistical analysis.

\section{Statistical Analysis}

The data were analyzed using SPSS 22.0 (IBM, Armonk, NY, USA). The continuous data were expressed as means \pm standard deviations and analyzed using Student's $t$-test. Categorical data were presented as frequencies and scores and were analyzed using Fisher's exact test. Multivariate linear regression (enter method) was used to analyze the factors associated with the standardized rehabilitation process. P-value $<0.05$ was considered statistically significant. 


\section{Results}

\section{Baseline Characteristics}

According to exclusion and inclusion criteria, among 453 parturients with DRA admitted at Xishan People's Hospital of Wuxi City between January 2017 and May 2021, a total of 294 patients were included in the study (Figure 2). Among them, 171 patients were treated with standardized rehabilitation (SR), and the other 123 patients were without SR treatment (non-SR). There was no statistically significant difference in age, weight, and BMI between these two groups ( $p>0.05$, Table 1). There was also no statistically significant difference in natural delivery, cesarean section, diabetes, hypertension, chronic constipation, abdominal pain, pelvic pain as well as low back pain between these two groups $((p>0.05$, Table 1$)$.

Before the treatment of standardized rehabilitation, there was no statistically significant difference between SR and non-SR in the measurement of the inner edges of the rectus abdominis, including the level of the center umbilicus and $4.5 \mathrm{~cm}$ above/below the umbilicus ( $p>0.05$, Table 2). However, compared with non-SR, the change in the inner edges of the rectus abdominis, including the level of the center umbilicus and $4.5 \mathrm{~cm}$ above/below the umbilicus, were obviously improved after the treatment with standardized rehabilitation in SR; the observed difference was statistically significant $(p<0.0001)$ (Tables 2 and 3$)$.

\section{Health-Related Quality of Life}

The Physical Functioning Scale (PFS-10) was used to evaluate the degree of mean values of health-related quality of life in physical functions; the list includes 10 items, as shown in Table 4. There was no statistically significant difference in the mean values of health-related quality of life before SR treatment between these two groups ( $p>0.05$, Table 4$)$.

After the treatment with standardized rehabilitation, and 20 days later, the Physical Functioning Scale (PFS10), which includes 10 items shown in Table 5, was used to evaluate the degree of mean values of health-related quality of life in physical functions. Compared with non$\mathrm{SR}$, mean values of health-related quality of life were obviously increased after the treatment of standardized rehabilitation in SR; there was no statistically significant difference $(p<0.0001$, Table 5).

All 294 postpartum women included in this study were followed up for 3 months at our hospital. For the parturient in SR, the symptoms of DRA did not recur or worsen. There were very few patients in non-SR whose DRA improved, even though not completely.

\section{Discussion}

DRA is one of the common complications of postpartum women. The treatment of diastasis rectus abdominis (DRA) can be complex. In the past, due to the lack of understanding of this disease, many patients could not get timely and effective diagnosis and treatment, which led to

Flow-chart of the standardized rehabilitation study

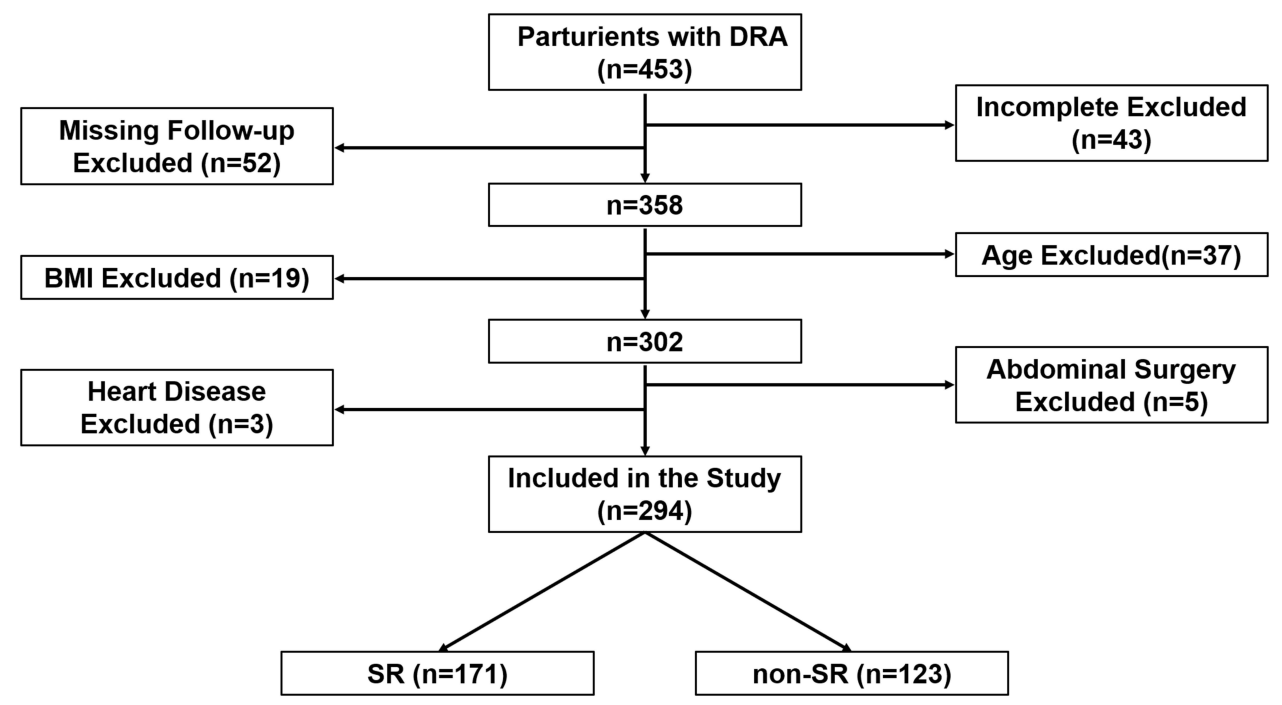

Figure 2 Flow-chart of the standardized rehabilitation study. The participants included in this study were selected based on the inclusion and exclusion criteria. 
Table I Characteristic and Clinical Features of Patients

\begin{tabular}{|l|c|c|c|}
\hline Items & SR (n=17I) & Non-SR (n=1 23) & P value \\
\hline Age (year) & $28.00 \pm 3.12$ & $27.35 \pm 3.33$ & 0.076 \\
\hline Weight $(\mathrm{kg})$ & $60.05 \pm 6.08$ & 60.1 I \pm 6.26 & 0.936 \\
\hline Numbers of pregnancies & $1.46 \pm 0.55$ & $1.44 \pm 0.56$ & 0.795 \\
\hline BMI (kg/m $)$ & $23.4 \pm 2.52$ & $23.47 \pm 2.43$ & 0.762 \\
\hline Natural delivery & 112 & 97 & 0.308 \\
\hline Cesarean section & 59 & 26 & 0.062 \\
\hline Diabetes & 5 & 3 & 0.906 \\
\hline Hypertension & 3 & 2 & 0.708 \\
\hline Chronic constipation & 31 & 3 & 0.502 \\
\hline Abdominal pain & 3 & 2 & 0.987 \\
\hline Pelvic pain & 4 & 66 & 1.000 \\
\hline Low back pain & 87 & 0.792 \\
\hline
\end{tabular}

Abbreviation: BMI, body mass index.

Table 2 The Inner Edges of the Rectus Abdominis Before and After Standardized Rehabilitation

\begin{tabular}{|c|c|c|c|c|c|c|}
\hline \multirow[t]{2}{*}{ Items } & \multicolumn{2}{|c|}{ Above the Umbilicus $(4.5 \mathrm{~cm})$} & \multicolumn{2}{|c|}{ Center of Umbilicus } & \multicolumn{2}{|c|}{ Below the Umbilicus $(4.5 \mathrm{~cm})$} \\
\hline & $\begin{array}{c}\text { Before SR } \\
\quad(\mathrm{cm})\end{array}$ & $\begin{array}{c}\text { After SR } \\
(\mathbf{c m})\end{array}$ & $\begin{array}{c}\text { Before SR } \\
\quad(\mathrm{cm})\end{array}$ & $\begin{array}{c}\text { After SR } \\
\text { (cm) }\end{array}$ & $\begin{array}{c}\text { Before SR } \\
\quad(\mathrm{cm})\end{array}$ & $\begin{array}{c}\text { After SR } \\
\text { (cm) }\end{array}$ \\
\hline$S R(n=|7|)$ & $4.58 \pm 0.66$ & $0.80 \pm 0.28$ & $4.62 \pm 0.81$ & $1.07 \pm 0.54$ & $4.4 I \pm 0.70$ & $0.96 \pm 0.37$ \\
\hline Non-SR $(n=123)$ & $4.48 \pm 1.02$ & $3.84 \pm 0.92$ & $4.68 \pm 0.49$ & $3.94 \pm 0.96$ & $4.36 \pm 0.92$ & $3.59 \pm 0.90$ \\
\hline$P$ value & 0.309 & $<0.0001$ & 0.466 & $<0.0001$ & 0.597 & $<0.0001$ \\
\hline
\end{tabular}

Table 3 Multiple Linear Regression Model (Before and After Standardized Rehabilitation)

\begin{tabular}{|l|c|c|c|c|c|c|c|c|c|}
\hline \multirow{2}{*}{ Items } & \multicolumn{2}{|c|}{ Above the Umbilicus (4.5cm) } & \multicolumn{2}{|c|}{ Center of Umbilicus } & \multicolumn{3}{|c|}{ Below the Umbilicus (4.5cm) } \\
\cline { 2 - 12 } & $\boldsymbol{\beta}$ & $\mathbf{t}$ & $\mathbf{P}$ value & $\boldsymbol{\beta}$ & $\mathbf{t}$ & $\mathbf{P}$ value & $\boldsymbol{\beta}$ & $\mathbf{t}$ & $\mathbf{P}$ value \\
\hline (constant) & 18.649 & 1.4 & 0.163 & 1.874 & 0.12 & 0.904 & 9.955 & 0.68 & 0.497 \\
\hline Age (year) & 0.025 & 1.919 & 0.056 & 0.03 & 2.026 & 0.044 & 0.02 & 1.419 & 0.157 \\
\hline Weight (Kg) & 0.146 & 1.331 & 0.184 & 0.02 & 0.155 & 0.877 & 0.081 & 0.67 & 0.503 \\
\hline Height (m) & -11.482 & -1.386 & 0.167 & -1.329 & -0.137 & 0.891 & -6 & -0.659 & 0.51 \\
\hline BMI (kg/m) & -0.389 & -1.37 & 0.172 & -0.044 & -0.133 & 0.894 & -0.216 & -0.69 & 0.491 \\
\hline Numbers of pregnancies & 0.029 & 0.415 & 0.678 & -0.004 & -0.055 & 0.956 & 0.052 & 0.691 & 0.49 \\
\hline Natural delivery vs Cesarean section & 0.126 & 1.459 & 0.146 & 0.082 & 0.812 & 0.417 & 0.105 & 1.108 & 0.269 \\
\hline SR vs non-SR & 2.493 & 32.55 & $<0.0001$ & 2.678 & 29.917 & $<0.0001$ & 1.934 & 22.978 & $<0.0001$ \\
\hline
\end{tabular}




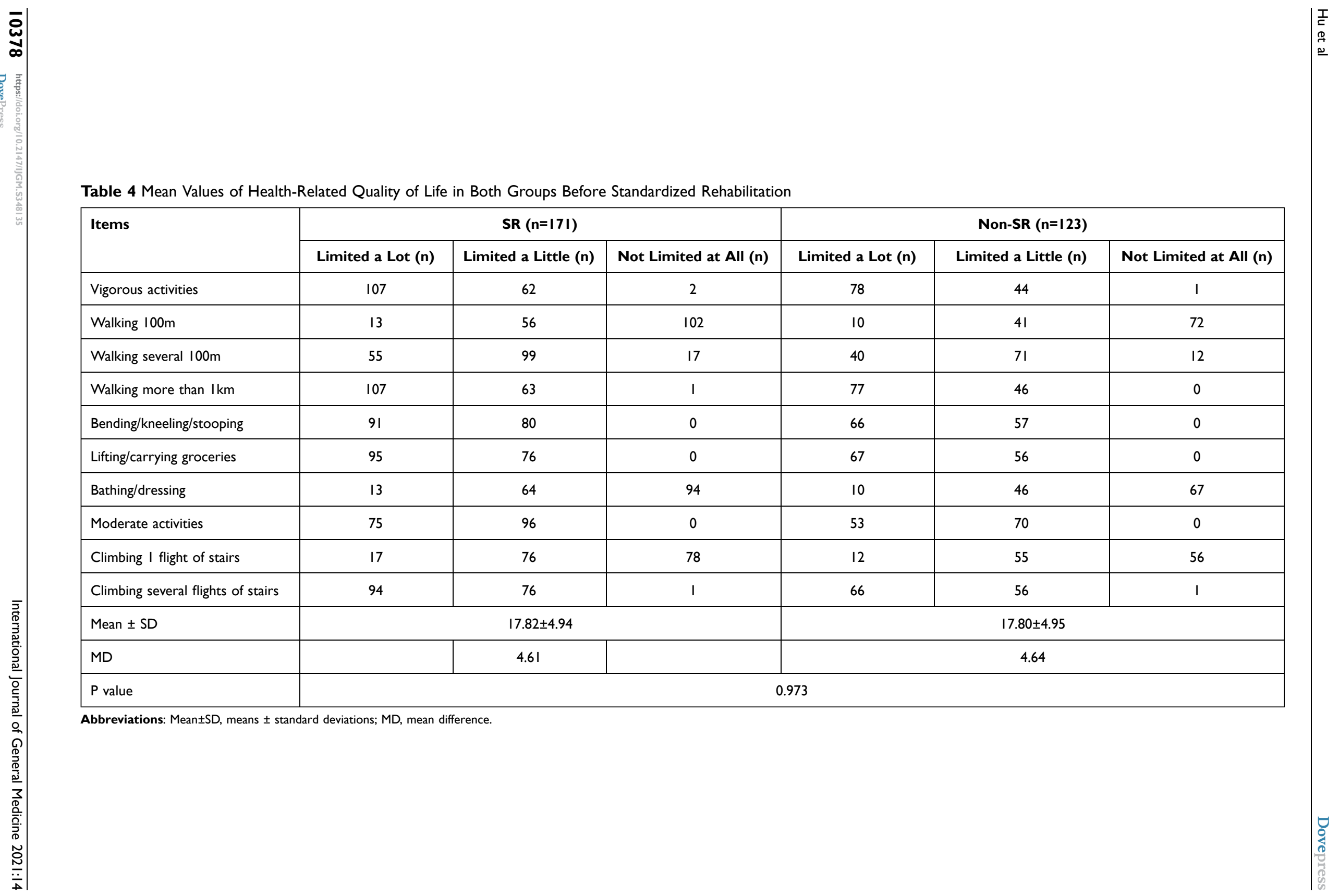


Table 5 Mean Values of Health-Related Quality of Life in Both Groups After Standardized Rehabilitation

\begin{tabular}{|c|c|c|c|c|c|c|}
\hline \multirow[t]{2}{*}{ Items } & \multicolumn{3}{|c|}{$S R(n=|7|)$} & \multicolumn{3}{|c|}{ Non-SR $(n=123)$} \\
\hline & Limited a Lot (n) & Limited a Little (n) & Not Limited at All (n) & Limited a Lot (n) & Limited a Little (n) & Not Limited at All (n) \\
\hline Vigorous activities & 50 & 44 & 77 & 73 & 48 & 2 \\
\hline Walking $100 \mathrm{~m}$ & 0 & 30 & 141 & 9 & 40 & 74 \\
\hline Walking several $100 \mathrm{~m}$ & 27 & 70 & 74 & 37 & 65 & 21 \\
\hline Walking more than $1 \mathrm{~km}$ & 55 & 53 & 63 & 71 & 46 & 6 \\
\hline Bending/kneeling/stooping & 56 & 40 & 75 & 64 & 53 & 6 \\
\hline Lifting/carrying groceries & 36 & 51 & 84 & 72 & 51 & 0 \\
\hline Bathing/dressing & 0 & 23 & 148 & 10 & 45 & 68 \\
\hline Moderate activities & 33 & 42 & 96 & 55 & 68 & 0 \\
\hline Climbing I flight of stairs & 0 & 48 & 123 & 11 & 53 & 59 \\
\hline Climbing several flights of stairs & 41 & 67 & 63 & 63 & 59 & I \\
\hline Mean \pm SD & \multicolumn{3}{|c|}{$23.78 \pm 6.25$} & \multicolumn{3}{|c|}{$|8.28 \pm 5.2|$} \\
\hline MD & \multicolumn{3}{|c|}{5.35} & \multicolumn{3}{|c|}{4.86} \\
\hline$P$ value & \multicolumn{6}{|c|}{$<0.0001$} \\
\hline
\end{tabular}

Abbreviations: Mean $\pm S D$, means \pm standard deviations; MD, mean difference. 
the continuous aggravation of DRA symptoms in the parturients and affected the quality of life. Early diagnosis and detection of DRA can avoid the occurrence and progressive aggravation of complications and the necessity of surgical treatment. Medical clinical centers are often the first-line facilities for diagnosing and treating DRA in postpartum women in China.

Herein, we designed standardized rehabilitation that can effectively alleviate the DRA of postpartum women (Tables 2-5). Based on the establishment of standardized rehabilitation (Figure S1-S19), normally an obstetriciangynecologist and a nurse-physical therapist together do the intervention and treatment for the patient in our center. During the latest period, from January 2017 to May 2021, a total of 294 patients were included in the study, there was no statistically significant difference between these two groups before the treatment of standardized rehabilitation (Table 1). In fact, the analysis of this study showed that the DRA separation (Tables 2 and 3) and the quality of life of postpartum women (Tables 4 and 5) was obviously improved after the early treatment of standardized rehabilitation. The Physical Functioning Scale (PFS-10) is a useful self-reported health measurement tool developed in the United States applying it to patients with acute and chronic diseases. $^{22}$ This study showed that the Mean values of health-related quality of life of DRA patients have been evidently improved after receiving standardized rehabilitation treatment (Tables 4 and 5). Importantly, those parturient treated by standardized rehabilitation, the symptoms of DRA did not recur or worsen with more than 3 months' follow-up.

In the past, little attention was paid to maternal postpartum DRA because of its mild early symptoms. However, with more and more researches and studies, a deeper understanding of DRA has been gained. ${ }^{12,25}$ DRA is one of the common complications during pregnancy and postpartum periods. It can appear around 14 weeks of pregnancy and gradually worsen until delivery. ${ }^{26}$ Although the condition itself has become clearer, diagnosis and treatment of DRA are still controversial. ${ }^{13,27}$ Previous studies have clearly reported that long-term postpartum DRA may lead to health complications, such as persistent low back pain, abdominal and pelvic pain, and similar. According to statistics, approximately $40 \%$ of women report persistent lower back and pelvic pain in the first half-year after childbirth. ${ }^{26,28}$ As a matter of fact, DRA is not a health problem for many postpartum women that can be solved by itself and may even progress for many years. ${ }^{29}$ Therefore, whether to intervene or provide treatment is related to the symptoms of DRA and whether they can be directly or indirectly relieved. The results of this study suggested that the DRA separation was significantly repaired and improved, including above the umbilicus $4.5 \mathrm{~cm}$, the Center of the umbilicus and below the umbilicus $4.5 \mathrm{~cm}$, not partial recovery (Tables 2 and 3). Early treatment and normalization of DRA separation is the key to improving the symptoms of patients. $^{20}$ This is also essential for improving the quality of life of postpartum women, as presented in this study (Tables 4 and 5). Concurrently, our study may also help other medical staff and rehabilitation physiotherapists engaged in postpartum rehabilitation in other clinical hospitals alleviate DRA symptoms and improve the patients' quality of life.

Previously, surgery has been considered an effective treatment for DRA. ${ }^{25}$ While depending on the understanding of DRA, non-surgical treatment and/or early active intervention methods are another effective way to treat DRA. ${ }^{13}$ Simultaneously, parturients regular abdominal exercises and aerobic exports, pelvic floor muscle exercise, posture and back care, corsets, acupuncture treatment, and other methods have all been recommended as efficient non-surgical interventions for DRA treatment. $^{2,13,16}$ The previous study has proven that abdominal muscle exercise is very effective in reducing DRA in the early postpartum period, which means exercise can significantly improve DRA symptoms and is an effective non-surgical solution. ${ }^{2}$ To date, there is little scientific knowledge on non-surgical methods recommended to treat DRA. Although there are many nonsurgical methods, there is also a lack of regulation and standardization. ${ }^{30}$ Herein, we set the standardized rehabilitation procedure (Figure S1-S19) and treated the patients since 2017, achieving effective results as presented above. All the parturients who received standardized rehabilitation treatment experienced significant improvement in their condition, and their quality of life was significantly improved.

In China, traditional acupuncture physiotherapy is efficient and has its own characteristics $;{ }^{16}$ however, it is very difficult to master. Using this method to treat DRA may also be time-consuming and laborious. Exercise pelvic floor muscles, rectus abdominis, and other exercise programs are mainly subjective recovery treatment methods, which require patients full perseverance to complete the exercise. $^{14}$ Other studies have reported that 
electrophysiological and magnetic therapy equipment can effectively relieve DRA in postpartum women. ${ }^{17}$ Based on the understanding of the previous research, combined with the characteristics of the electrophysiological treatment, we designed the current standardized rehabilitation treatment program for DRA (Figure S1-S19), which has been promoted through clinical trials over recent years, with good effects. This suggested that standardized rehabilitation treatment is safe and efficient for parturients with DRA. The standardized rehabilitation treatment model we set mainly includes the standardized operation of medical staff or rehabilitation physiotherapists and the proficient use of electrophysiological treatment equipment. The whole course of treatment has a short cycle (20 days,

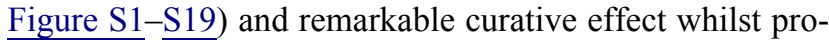
viding patients with a comfortable treatment environment (Tables 4 and 5). Patients could better cooperate with the medical staff and/or rehabilitation physiotherapists within the standardized rehabilitation treatment. Furthermore, compared with other non-surgical treatments for DRA, standardized rehabilitation treatment significantly improved the separation of the rectus abdominis above the umbilical cord, below the umbilical cord, and the central part of the umbilicus of the parturients' DRA, evading partial recovery.

\section{Clinical Implication}

The non-surgical treatment used for DRA is somewhat different from the standardized rehabilitation treatment we designed. The current research plan adopts a combination of Chinese and Western medicine, which is more convenient to master, apply and promote. Our research provides a simple and effective standardized method to effectively treat patients with DRA. We hope to promote this standardized rehabilitation treatment, as well as to obtain further improvements and enhancements.

\section{Conclusion}

The purpose of our design and establishment of standardized rehabilitation treatment is to help parturients with DRA. This retrospective study revealed that our standardized rehabilitation treatment method is simplicity and efficiency. This approach can improve the quality of life of parturients, thus could be further promoted and applied.

\section{Data Sharing Statement}

The datasets used and/or analyzed during the current study are available from the corresponding author, Chaobo
Chen, on reasonable request. For any queries, kindly contact bobo19820106@gmail.com.

\section{Ethics Approval and Consent to Participate}

The study was approved by the Ethics Committee of Xishan People's Hospital of Wuxi City, No. xs2020ky013. The need for individual consent was waived by the committee. This article was a retrospective study, patient parental consent to review their medical records was not required by the ethics Committee of Xishan People's Hospital of Wuxi City. This study strictly kept the patients' information confidential. The study complied with the Declaration of Helsinki.

\section{Acknowledgments}

The authors thank all the members of the Department of Postpartum Rehabilitation Center and Department of General Surgery, Wuxi Xishan People's Hospital, for their persevering work.

\section{Author Contributions}

All authors have read and approved the final manuscript; have made important contributions to the work of the report, whether in terms of methods creation, research design, implementation, data aggregation, statistical analysis, and interpretation, etc., and participated in drafting, revising, and reviewing the manuscript; finally reaching an agreement on the journal to which the article was submitted; and finally agreed to be responsible for all aspects of the work.

Jiang $\mathrm{Hu}$ and Juanfen $\mathrm{Gu}$ have contributed equally as co-first authors. Chaobo Chen and Lina You should be considered joint corresponding authors.

\section{Funding}

This research was supported by the Wuxi Municipal Health Commission's Maternal and Child Health Appropriate Technology Promotion (Project, No. FYTG201908).

\section{Disclosure}

The authors declare no conflicts of interest for this work.

\section{References}

1. Laframboise FC, Schlaff RA, Baruth M. Postpartum exercise intervention targeting diastasis recti abdominis. Int J Exerc Sci. 2021;14 (3):400-409. 
2. Thabet AA, Alshehri MA. Efficacy of deep core stability exercise program in postpartum women with diastasis recti abdominis: a randomised controlled trial. $J$ Musculoskelet Neuronal Interact. 2019;19(1):62-68.

3. Fernandes da Mota PG, Pascoal AG, Carita AI, Bo K. Prevalence and risk factors of diastasis recti abdominis from late pregnancy to 6 months postpartum, and relationship with lumbo-pelvic pain. Man Ther. 2015;20(1):200-205. doi:10.1016/j.math.2014.09.002

4. Gitta S, Magyar Z, Tardi P, et al. [Prevalence, potential risk factors and sequelae of diastasis recti abdominis]. Orv Hetil. 2017;158 (12):454-460. Hu. doi:10.1556/650.2017.30703

5. Kimmich N, Haslinger C, Kreft M, Zimmermann R. [Diastasis Recti Abdominis and Pregnancy]. Praxis. 2015;104(15):803-806. German. doi:10.1024/1661-8157/a002075

6. Wu L, Gu Y, Gu Y, et al. Diastasis recti abdominis in adult women based on abdominal computed tomography imaging: prevalence, risk factors and its impact on life. J Clin Nurs. 2021;30(3-4):518-527. doi:10.1111/jocn. 15568

7. Gluppe SL, Hilde G, Tennfjord MK, Engh ME, Bo K. Effect of a postpartum training program on the prevalence of diastasis recti abdominis in postpartum primiparous women: a randomized controlled trial. Phys Ther. 2018;98(4):260-268. doi:10.1093/ptj/pzy008

8. Mayer HF, Loustau HD. The suprapubic dermoadipose flap for aesthetic reshaping of the postpregnancy abdomen. Aesthet Surg J. 2018;38(6):635-643. doi:10.1093/asj/sjx175

9. Benjamin DR, Frawley HC, Shields N, van de Water ATM, Taylor NF. Relationship between diastasis of the rectus abdominis muscle (DRAM) and musculoskeletal dysfunctions, pain and quality of life: a systematic review. Physiotherapy. 2019;105(1):24-34. doi:10.1016/j.physio.2018.07.002

10. Emanuelsson P, Gunnarsson U, Dahlstrand U, Strigard K, Stark B. Operative correction of abdominal rectus diastasis (ARD) reduces pain and improves abdominal wall muscle strength: a randomized, prospective trial comparing retromuscular mesh repair to double-row, self-retaining sutures. Surgery. 2016;160(5):1367-1375. doi:10.1016/ j.surg.2016.05.035

11. Mommers EHH, Ponten JEH, Al Omar AK, de Vries Reilingh TS, Bouvy ND, Nienhuijs SW. The general surgeon's perspective of rectus diastasis. A systematic review of treatment options. Surg Endosc. 2017;31(12):4934-4949. doi:10.1007/s00464-017-5607-9

12. Keshwani N, Mathur S, McLean L. The impact of exercise therapy and abdominal binding in the management of diastasis recti abdominis in the early post-partum period: a pilot randomized controlled trial. Physiother Theory Pract. 2021;37(9):1018-1033. doi:10.1080/ 09593985.2019.1675207

13. Gluppe SB, Engh ME, Bo K. Immediate effect of abdominal and pelvic floor muscle exercises on interrecti distance in women with diastasis recti abdominis who were parous. Phys Ther. 2020;100 (8):1372-1383. doi:10.1093/ptj/pzaa070

14. Keshwani N, Mathur S, McLean L. The impact of exercise therapy and abdominal binding in the management of diastasis recti abdominis in the early post-partum period: a pilot randomized controlled trial. Physiother Theory Pract. 2019;37:1-16.

15. Kamel DM, Yousif AM. Neuromuscular electrical stimulation and strength recovery of postnatal diastasis recti abdominis muscles. Ann Rehabil Med. 2017;41(3):465-474. doi:10.5535/arm.2017.41.3.465

16. Elden H, Ostgaard HC, Fagevik-Olsen M, Ladfors L, Hagberg H. Treatments of pelvic girdle pain in pregnant women: adverse effects of standard treatment, acupuncture and stabilising exercises on the pregnancy, mother, delivery and the fetus/neonate. BMC Complement Altern Med. 2008;8(1):34. doi:10.1186/1472-6882-8-34
17. Kinney BM, Lozanova P. High intensity focused electromagnetic therapy evaluated by magnetic resonance imaging: safety and efficacy study of a dual tissue effect based non-invasive abdominal body shaping. Lasers Surg Med. 2019;51(1):40-46. doi:10.1002/1sm.23024

18. Urits I, Schwartz RH, Orhurhu V, et al. A comprehensive review of alternative therapies for the management of chronic pain patients: acupuncture, tai chi, osteopathic manipulative medicine, and chiropractic care. Adv Ther. 2021;38(1):76-89. doi:10.1007/s12325-02001554-0

19. Albaladejo-Belmonte M, Tarazona-Motes M, Nohales-Alfonso FJ, De-arriba M, Alberola-Rubio J, Garcia-Casado J. Characterization of pelvic floor activity in healthy subjects and with chronic pelvic pain: diagnostic potential of surface electromyography. Sensors (Basel. 2021;21(6):2225.

20. Ptaszkowska L, Gorecka J, Paprocka-Borowicz M, et al. Immediate effects of kinesio taping on rectus abdominis diastasis in postpartum women-preliminary report. $J$ Clin Med. 2021;10(21):5043. doi: $10.3390 / \mathrm{jcm} 10215043$

21. Cardaillac C, Vieillefosse S, Oppenheimer A, Joueidi Y, Thubert T, Deffieux X. Diastasis of the rectus abdominis muscles in postpartum: concordance of patient and clinician evaluations, prevalence, associated pelvic floor symptoms and quality of life. Eur $J$ Obstet Gynecol Reprod Biol. 2020;252:228-232. doi:10.1016/j. ejogrb.2020.06.038

22. Ware JE Jr, Sherbourne CD. The MOS 36-item short-form health survey (SF-36). I. Conceptual framework and item selection. Med Care. 1992;30(6):473-483. doi:10.1097/00005650-199206000-00002

23. McHorney CA, Ware JE Jr, Raczek AE. The MOS 36-Item Short-Form Health Survey (SF-36): II. Psychometric and clinical tests of validity in measuring physical and mental health constructs. Med Care. 1993;31(3):247-263. doi:10.1097/00005650-19930300000006

24. Bohannon RW, DePasquale L. Physical Functioning Scale of the Short-Form (SF) 36: internal consistency and validity with older adults. J Geriatr Phys Ther. 2010;33(1):16-18.

25. Malcher F, Lima DL, Lima R, et al. Endoscopic onlay repair for ventral hernia and rectus abdominis diastasis repair: why so many different names for the same procedure? A qualitative systematic review. Surg Endosc. 2021;35(10):5414-5421. doi:10.1007/s00464021-08560-5

26. Sperstad JB, Tennfjord MK, Hilde G, Ellstrom-Engh M, Bo K. Diastasis recti abdominis during pregnancy and 12 months after childbirth: prevalence, risk factors and report of lumbopelvic pain. Br J Sports Med. 2016;50(17):1092-1096. doi:10.1136/bjsports2016-096065

27. van de Water AT, Benjamin DR. Measurement methods to assess diastasis of the rectus abdominis muscle (DRAM): a systematic review of their measurement properties and meta-analytic reliability generalisation. Man Ther. 2016;21:41-53. doi:10.1016/j. math.2015.09.013

28. Parker MA, Millar LA, Dugan SA. Diastasis rectus abdominis and lumbo-pelvic pain and dysfunction-Are they related? Journal of Women's Health Physical Therapy. 2009;33(2):15-22. doi:10.1097/ 01274882-200933020-00003

29. Ranney B. Diastasis recti and umbilical hernia causes, recognition and repair. $S$ D J Med. 1990;43(10):5-8.

30. Carlstedt A, Bringman S, Egberth M, et al. Management of diastasis of the rectus abdominis muscles: recommendations for Swedish National Guidelines. Scand J Surg. 2020:1457496920961000. doi: $10.1177 / 1457496920961000$ 


\section{Publish your work in this journal}

The International Journal of General Medicine is an international, peer-reviewed open-access journal that focuses on general and internal medicine, pathogenesis, epidemiology, diagnosis, monitoring and treatment protocols. The journal is characterized by the rapid reporting of reviews, original research and clinical studies across all disease areas. The manuscript management system is completely online and includes a very quick and fair peer-review system, which is all easy to use. Visit http://www.dovepress.com/ testimonials.php to read real quotes from published authors.

Submit your manuscript here: https://www.dovepress.com/international-journal-of-general-medicine-journal 